\title{
Tuberous sclerosis complex: A case report and literature review
}

\author{
Amjad Ali1", Dipankar Das², Harsha Bhattacharjee ${ }^{3}$, Damaris Magdalene ${ }^{4}$, Divakant Misra ${ }^{5}$ \\ ${ }^{1}$ Comprehensive Ophthalmology Fellow, ${ }^{2}$ Consultant, ${ }^{3}$ Senior Consultant and Managing Director, ${ }^{4}$ Consultant, ${ }^{5}$ Vitreo-Retinal \\ Fellow, ${ }^{1,2,3}$ Dept. of Ocular Pathology, ${ }^{4}$ Dept. of Paediatric Ophthalmology, ${ }^{5}$ Dept. of Vitreo-Retina Surgery, Sri Sankaradeva \\ Nethralaya, Guwahati, Assam, India
}

*Corresponding Author:

Email: ali.leo.amjad@gmail.com

\begin{abstract}
Tuberous Sclerosis Complex is a rare genetic disorder of autosomal dominant inheritance. It is a neurocutaneous syndrome exhibiting multiple hamartomatous proliferations involving multiple organ system such as brain, kidney, heart, lungs, eyes and skin. Here, we present a case report of a 7year old male patient with characteristic clinical and radiological features of Tuberosis Sclerosis Complex.
\end{abstract}

Keywords: Genetic disorder, Multiple hamartomas, Neurocutaneous syndrome, Tuberous sclerosis complex.

\section{Case Report}

A 7 year old male patient presented to the outpatient department for ophthalmic evaluation. He was a diagnosed case of epilepsy with first episode of seizure at 6months of age and had been on antiepileptic medications (Tab vigabatrin $250 \mathrm{mg}$ ) since then. Last epileptic episode was 1year back. The patient was mentally retarded with learning difficulties, for which he was undergoing speech therapy and physiotherapy. Birth history and family history were insignificant.
History of delayed developmental milestones was present.

On general physical examination, multiple flat, reddish macular lesions were noted on the nose and cheeks in a characteristic "butterfly pattern", suggestive of Adenoma sebaceum (Fig. 1A). An irregular, hypo pigmented patch was seen on the trunk, indicative of ash leaf macule (Fig. 1B). A fibrous plaque on the chin (Fig. 1C) and clubbing of fingers was also noted. Shortness of breath and subungual hamartoma were absent.
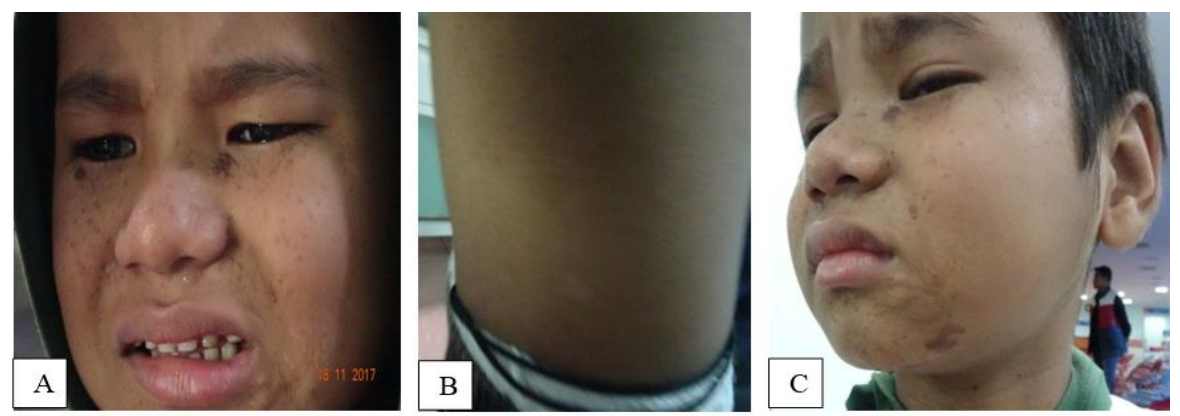

Fig. 1: Clinical photograph of the patient showing; (A) Adenoma sebaceum- flat, reddish macular lesions with a butterfly distribution around the nose and cheeks; (B) Ash leaf macules on trunk; (C) Fibrous plaques

On ocular examination, a central, steady and maintained corneal reflex was present. Adnexa and anterior segment of both eyes was normal. Examination under anaesthesia of right eye revealed a single, flat, smooth surfaced, non-calcified and translucent lesion in the superotemporal part of retina with adjoining sclerosed vessels, suggestive of type 2 retinal hamartoma. Fundus of left eye was normal.

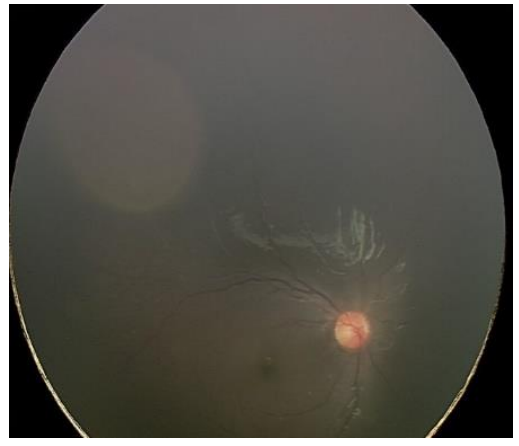

Fig. 2: Fundus phohotograph of right eye showing flat no calcified translucent lesion in the superotemporal part of retina (Retinal hamartoma) with sclerosed vessels 
Magnetic resonance imaging (MRI) of brain revealed multiple T2 weighted/ Short tau inversion recovery (STIR) hyperintense and $\mathrm{T} 1$ weighted isointense lesions suggestive of cortical and subcortical tubers in frontal, temporal and parieto-occipital lobes. Multiple calcified subependymal nodules were seen on either side evident by increased signal on $\mathrm{T} 1$ and signal dropout on Gradient echo sequences (GRE). Periventricular white matter paucity was noted on either side with T2 weighted/STIR hyperintense white matter radial band on right side. ${ }^{14}$
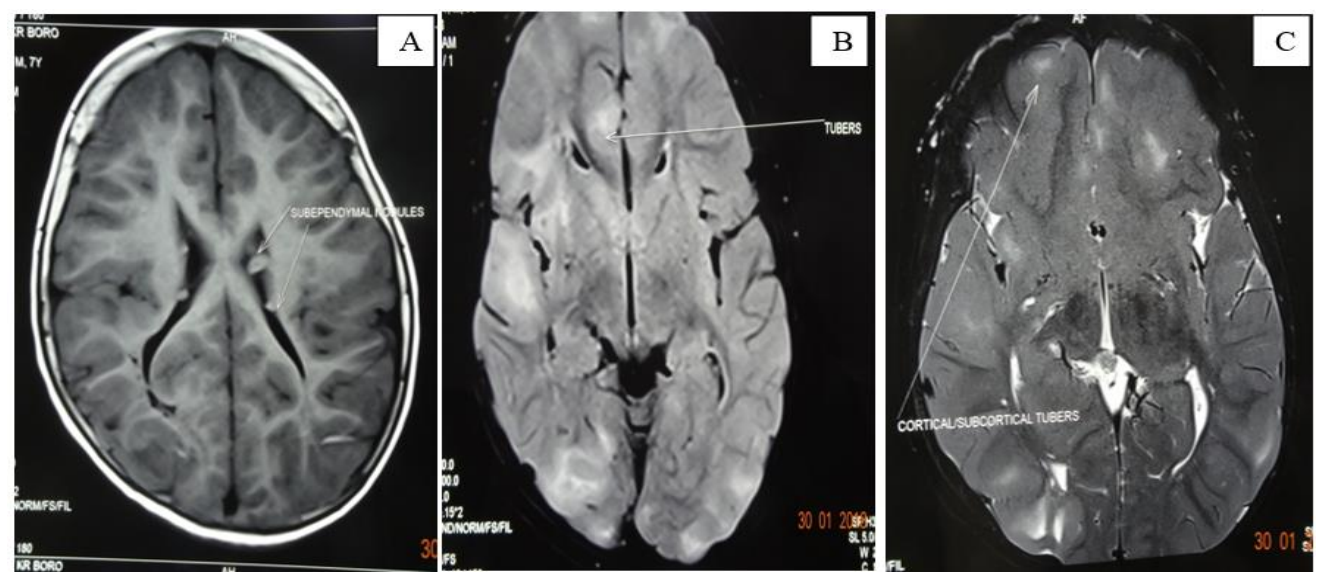

Fig. 3: T1 weighted magnetic resonance images showing (A) Multiple subependymal nodules (B, C) Cortical and suncortical tubers

High-resolution computed tomography (HRCT) of the thorax and abdomen showed no abnormality. Flash visually evoked potentials (VEP's) were markedly prolonged with normal amplitudes on both sides evident of bilateral disturbance in visual pathways. Electroencephalography (EEG) was abnormal due to predominantly left fronto-polar as well as bisynchronous temporo-occipital inter-ictal epileptic discharges.

Based on all the clinical findings and investigations, a final diagnosis of Tuberous sclerosis was confirmed.

\section{Discussion}

Tuberous sclerosis complex (TSC) is a rare neurocutaneous syndrome which variably affects the brain, kidney, heart, lungs, eyes and skin. It is characterized by neuropsychological manifestations, such as seizures, autism and cognitive disability and the formation of unusual tumour-like growths (hamartomas) in more than one organ system. ${ }^{1}$

TSC was first described by von Recklinghausen in 1862 and later elaborated by a French neurologist Desire-Magloire Bourneville in 1880, henceforth also known as Bourneville's disease. In 1920 Van der Hoeve first recognized retinal involvement in tuberous sclerosis. It is an autosomal dominant disorder, caused by mutations of either the TSC1 gene on chromosome 9q34 encoding hamartin or the TSC2 gene on chromosome $16 \mathrm{p} 13$ encoding tuberin. These two genes together form a tumour suppressor complex, which inhibit the mammalian target of rapamycin (mTOR), an evolutionarily conserved protein kinase and a major effector of cell growth. ${ }^{10}$ The mutation in these genes leads to mTOR activation and formation of various growths and hamartomas in various organs of the body. ${ }^{2,15}$

The frequency of TSC is estimated to be $1 / 6000$ to $1 / 10,000$ live births and a prevalence of around $1 / 20,000 .{ }^{3}$ TSC has clinical manifestations in different organ systems that continue to develop over the lifetime of an affected individual. ${ }^{12}$

The clinical features of tuberous sclerosis are highly variable, hence making the diagnosis of the disease very challenging. The classic Vogt's triad which included seizures, mental retardation, and cutaneous angiofibromas, occurs in only $29 \%$ of cases. The diagnosis is based on the TSC diagnostic criteria (recently revised in 2012) laid down by the tuberous sclerosis complex consensus group (Table 1).

\section{Table 1: Updated diagnostic criteria for tuberous} sclerosis complex $2012^{4}$

\begin{tabular}{l} 
A. Genetic diagnostic criteria \\
The identification of either a TSC1 or TSC2 \\
pathogenic mutation in DNA from normal tissue is \\
sufficient to make a definite diagnosis of tuberous \\
sclerosis complex (TSC). A pathogenic mutation is \\
defined as a mutation that clearly inactivates the \\
function of the TSC1 or TSC2 proteins (e.g., out- \\
of-frame indel or nonsense mutation), prevents \\
protein synthesis (e.g., large genomic deletion), or \\
is a missense mutation whose effect on protein \\
function has been established by functional \\
assessment. Other TSC1 or TSC2 variants whose \\
effect on function is less certain do not meet these \\
criteria, and are not sufficient to make a definite \\
diagnosis of TSC. Note that $10 \%$ to $25 \%$ of TSC \\
patients have no mutation identified by \\
\hline
\end{tabular}


conventional genetic testing, and a normal result does not exclude TSC, or have any effect on the use of clinical diagnostic criteria to diagnose TSC.

\section{B. Clinical diagnostic criteria \\ Major features}

1. Hypomelanotic macules $(\geq 3$, at least $5-\mathrm{mm}$ diameter)

2. Angiofibromas $(\geq 3)$ or fibrous cephalic plaque

3. Ungual fibromas $(\geq 2)$

4. Shagreen patch

5. Multiple retinal hamartomas

6. Cortical dysplasias*

7. Subependymal nodules

8. Subependymal giant cell astrocytoma

9. Cardiac rhabdomyoma

10. Lymphangioleiomyomatosis (LAM)**

11. Angiomyolipomas $(\geq 2)$

Minor features

1. "Confetti" skin lesions

2. Dental enamel pits $(\geq 3)$ [5]

3. Intraoral fibromas $(\geq 2)$

4. Retinal achromic patch

5. Multiple renal cysts

6. Nonrenal hamartomas

\section{Definite diagnosis:}

Two major features or one major feature with $\geq 2$ minor features

\section{Possible diagnosis:}

Either one major feature or $\geq 2$ minor features

* Includes tubers and cerebral white matter radial migration lines.

** A combination of the two major clinical features (LAM and angiomyolipomas) without other features does not meet criteria for a definite diagnosis

Ophthalmic Manifestations: About 50\% of the cases with Tuberous sclerosis have ocular involvement; henceforth the role of an ophthalmologist in early diagnosis of the disease is crucial.

The pathognomic retinal lesions of tuberous sclerosis are astrocytic hamartomas. They are seen in about $53 \%$ of the patients. Tuberous sclerosis typically presents with multifocal or bilateral hamartomas, as compared to solitary hamartomas occasionally present in otherwise healthy individuals. Astrocytic hamartomas can be morphologically classified into two types: (1) Large, whitish (calcified) nodular masses or (2) Flat, translucent (noncalcified) smooth tumours. ${ }^{11}$ An intermediate type of retinal hamartoma having features of both types has also been described. ${ }^{6}$ Rarely retinal hamartomas may be associated with complications such as vitreous hemorrhage, retinal vascular abnormalities (including telangiectasia, neovascularization, and exudation), and vitreous seeding.
Some patients may also present with astrocytomas involving the optic nerve. Optic nerve astrocytomas may initially have a greyish or greyish pink appearance, but later develop a glistening, yellow, mulberry appearance. They may cause vitreous seeding and vitreous hemorrhage. In the presence of a single retinal (or optic nerve) hamartoma, only one additional major feature, or two or more minor features, are required to make the diagnosis of tuberous sclerosis complex.

The first step for the ophthalmologist should be, to carefully re-examine both eyes for signs of one or more retinal or optic nerve hamartomas. Retinal and optic nerve astrocytic hamartomas can also be present in patients with neurofibromatosis. If the diagnosis of tuberous sclerosis or neurofibromatosis cannot be clearly established, the appearance of the retinal or optic nerve hamartoma may provide clinical clues related to the associated systemic condition. Shields and Shields state that a tumor that consists of a mulberrylike calcification is pathogonomonic of tuberous sclerosis. However, when the only retinal tumour seen is a small, translucent, and whitish noncalcified thickening of the nerve fiber layer, it may be difficult, if not impossible, to distinguish it from myelinated nerve fibers or retinoblastoma.

Non- retinal signs include angiofibromas (adenoma sebaceum) of the lids, giving rise to a typical salmon colored lid, poliosis of eyelashes and hypopigmented iris spots which may be an early sign of the disease. ${ }^{7}$ Refractive errors may be present in $20-30 \%$ cases.

\section{Conclusion}

Nearly one million people are estimated to have TSC worldwide. Due to the relative obscurity of the disease and the mild form symptoms, many cases may remain undiagnosed for years or decade. Early diagnosis and intervention can help overcome developmental delays and behavioural abnormalities. Early seizure control in children can improve learning as compared to children without good seizure control. Tuberous sclerosis is a lifelong condition that requires long-term multidisciplinary approach:

1. Ophthalmologist may play an important role in early diagnosis, as the characteristic retinal hamartomas can often be detected within the first two years of life.8

2. Paediatric psychiatrists can advise on pharmacologic management of behavioural problems.

3. Neurosurgeons can assist in the placement of a vagus nerve stimulator and assess the patient as a candidate for corpus callosotomy or focal resection, in special cases. ${ }^{13}$

4. Nephrologist's opinion is necessary for individuals with polycystic kidney disease, AMLs, or endstage renal disease.

5. Paediatric neuropsychologists can assess intellectual function and educational needs and 
advise on non-pharmacologic management of behavioural problems. Because children with TSC are at developmental risk, neuropsychological assessment is recommended at diagnosis and prior to entering school.

6. Pulmonary medicine consultation is necessary for individuals with LAM, pneumothorax, or other types of lung involvement.

7. Dieticians can help in the institution and maintenance of the ketogenic diet. ${ }^{9}$

Although there is no specific cure for tuberous sclerosis, early diagnosis and symptomatic management may improve the quality of life of such patients. Currently in addition to FDA approval for renal angiolipomas and subependymal astrocytomas that cannot be resected, mTORC inhibitors (like rapamycin or everolimus) are in clinical trials for TSC-related refractory epilepsy, neurocognitive manifestations, and facial angiofibromas. ${ }^{16}$

Acknowledgment: We would like to thank Sri Kanchi Sankara Health and Educational Foundation, Guwahati, India.

Declaration of Patient Consent: The authors certify that they have obtained all appropriate patient consent forms. In the form the patient(s) has/have given his/her/their consent for his/her/their images and other clinical information to be reported in the journal. The patients understand that their names and initials will not be published and due efforts will be made to conceal their identity, but anonymity cannot be guaranteed.

\section{Financial Support and Sponsorship: Nil.}

Conflicts of Interest: There are no conflicts of interest.

\section{References}

1. Sarkar S, Khaitan T, Sinha R, Kabiraj A. Tuberous sclerosis complex: a case report. Contemp Clin Dent. 2016;7(2):236-239.

2. Au KS, Williams AT, Roach ES, Batchelor L, Sparagana SP, Delgado MR. Genotype/phenotype correlation in 325 individuals referred for a diagnosis of tuberous sclerosis complex in the United States. Genet Med. 2007;9(2):88100.

3. Northrup H, Krueger DA. Tuberous sclerosis complex diagnostic criteria update: recommendations of 012 international tuberous sclerosis complex consensus conference. Paediatr Neurol. 2013;49(4):243-54.

4. Krueger DA, Northup H. The International Tuberous Sclerosis Consensus Group. Tuberous Sclerosis Complex Surveillance and Management: Recommendations of the 2012 International Tuberous Sclerosis Complex Consensus Conference. Paediatr Neurol. 2013;49(4):255265.

5. Sampson JR, Attwood D, Al Mughery AS, Reid JS. Pitted enamel hypoplasia in tuberous sclerosis. Clin genect. 1992;42(1):50-52.
6. Nyboer JH, Robertson DM, Gomez MR. Retinal lesions in tuberous sclerosis. Arch Ophthalmol. 1976;94(8):1277-1280.

7. Gutman I, et al. Hypopigmented iris spots: an early sign of tuberous sclerosis. Ophthalmology. 1982;89(10):11551159.

8. Grover WD, Harley RD. Early recognition of tuberous sclerosis by funduscopic examination. $J$ Pediatr. 1969;75(6):991-995.

9. The effect of ketogenic diet in refractory partial seizures with reference to tuberous sclerosis. Eur J Paediatr Neur. 2006;10(3):148-51.

10. Northup H, Koenig M, Au K. Tuberous sclerosis complex. Genereview.2011; http://www.genetests.org

11. Aldrich CS, Hong CH, Groves L, Olsen C, Moss J, Darling TN. Acral lesions in tuberous sclerosis complex: insight into pathogenesi. J Am Acad Dermatol. 2010;63(2):244-251.

12. Verna B, Tailor M. Familial tuberous sclerosis: a review with report of three cases. Indian Pediatr. 1965;2(11):401-410.

13. Fallah A, Rodgers SD, Weil AG, et al. Resective epilepsy surgery for tuberous sclerosis in childrens: determining predictors of seizures outcomes in a multicenter retrospective cohort study. Neurosurgery. 2015;77(4):517-524.

14. Roach ES, Williams DP, Laster DW. Magnetic resonance imaging in tuberous sclerosis. Arch Neurol. 1987;44(3):301-303.

15. Curatolo $P$, Mechanistic target of rapamycin (mTOR) in tuberous sclerosis complex-asociated epilepsy. Paediatr Neurol. 2015;52(3):281-289.

16. Dilli PE, De Bernardis G, Weber P, Losch U. Topical Everolimus for facial angiofibromas in the tuberous sclerosis. A first case report. Pediatr Neurol. 2014;51(1):109-113.

How to cite this article: Ali $A$, Das D, Bhattacharjee H, Magdalene D, Misra D. Tuberous sclerosis complex: A case report and literature review. Ind J Clin Exp Ophthalmol. 2018;4(3):290-293. 